\title{
EFFICIENCY ESTIMATION OF BEAM EXTRACTION BY THE SCATTERING TARGET
}

\section{I.I.Degtyarev, I.A.Yazynin, SRC Institute for High Energy Physics, Protvino, Russia}

E-mail: yazynin@mx.ihep.su

\section{Abstract}

The possibility of using the scattering targets for forming and extraction beam in the modern circular accelerators is considered in paper. Is shown that the minimum losses of particles will be by use of targets from heavy elements such as tungsten. The optimum sizes of a target, its displacement from septum and frequency of the betatron oscillations are determined.

The analytical and numerical accounts show, that at a beam extraction of energy $70 \mathrm{GeV}$ from $\mathrm{U} 70$ and 600 $\mathrm{GeV}$ from $\mathrm{U} 600$ is possible to reach efficiency $\sim 70 \%$ and $\sim 90 \%$ accordingly.

\section{Introduction}

The use of amorphous target as a scatter of charged particles is widely applied in circular accelerators (IHEP, JINR, DESY, FNAL, CERN) /1,2/ for the forming a beam, localization of losses and beam extraction. A main problem arising at the designing of such systems is the decrease of inevitable losses of particles on the scattering target, on the septum or deflector partition, on the collimators and vacuum chambers.

With increase of beam energy the efficiency of considered extraction beam systems because of reducing of root-mean-square magnitude of angular scattering of particles on the target $\sigma \sim 1 / \gamma$ considerably may decrease. With increase of beam intensity and with use of superconductor magnetic elements the solution of localization problem of the inevitable losses becomes complicated. There is a probability of the overheating and destruction the target and significant radiation of the neighboring magnetic equipment and the problem of using possibility such systems is put.

In work the choice and account of optimum parameters of the scattering target from the viewpoint of losses minimization is considered. The advantage of use the targets from heavy elements such as tungsten are proved. The numerical simulation of beam extraction from the UNK and U70 is made.

\section{The beam extraction of charged particles on the amorphous target}

The layout of the beam extraction system with use of the amorphous target is shown on Fig.1. The beam by the bump - magnet is guided to the target which executed as a slice standing across a beam. At the interaction with substance atoms the particle in main due to the multiple Coulomb scattering change the amplitude of betatron oscillations and such have a possibility through some turns in the accelerator to hit in the septum - magnet clearance and to send in the extraction channel.

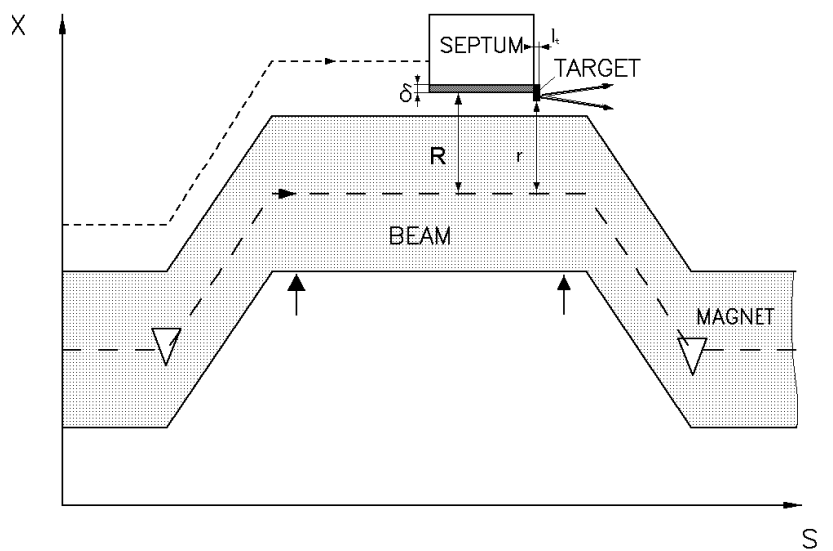

Fig.1: The extraction system layout.

At the extraction the particles are lost on the target due to the nuclear interactions $\eta_{t}$, some hit on the septum partition $\eta_{s}$, and the part of protons scattered on the large angles hits on the equipment standing on a beam course behind the target $\eta_{a}$. That is the total magnitude of losses can be defined $\eta=\eta_{t}+\eta_{s}+\eta_{a}$. The extraction efficiency depends on the beam parameters, structure, substance of the target, its sizes and position, effective thickness of the septum. Determination of conditions, at which the losses will be minimum, is the purpose of the given work.

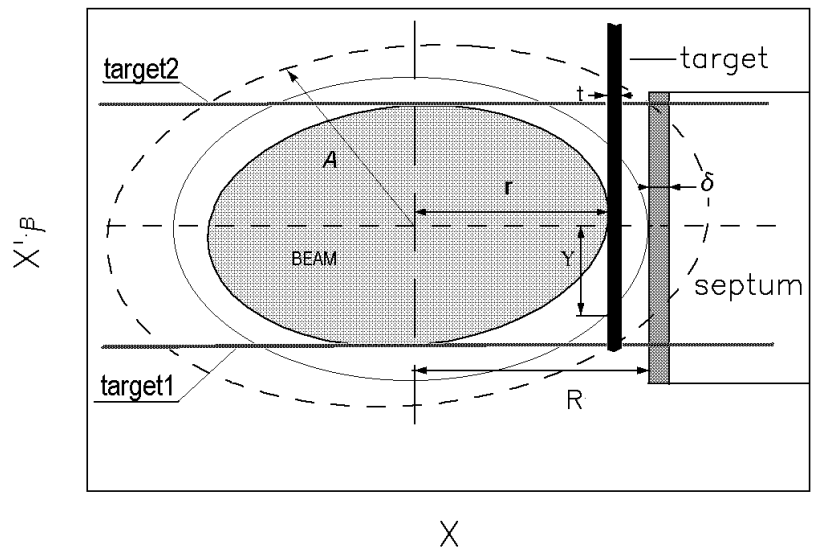

Fig.2: The extraction phase space.

At the extraction of a beam on the scattering target we have a diffusion process of amplitude growth the beam particles with boundary conditions. At the magnitude of amplitude $A>R$ the particles can hit on the septum. For the target, which has small transversal size $t$, the average passages of the particle through it can be estimated: $\bar{N}=1+k\left(\frac{y}{D}\right)^{m}$. Where $y=\sqrt{R^{2}-r^{2}}$ is the coordinate of the such point (Fig.2), that if particle is farther than it, 
it hit on the septum in main, and $r=r_{t} \sqrt{\beta_{s} / \beta_{t}}, r_{t}$ - distance of the target edge from the beam axis, $D=\sigma \beta, \sigma$ and $D$ are the r.m.s. magnitude of scattering on the target on angle and coordinate accordingly, $\beta=\sqrt{\beta_{t} \beta_{s}}, \beta_{t}, \beta_{s}$ is the amplitude functions in the location of the target and septum. Magnitude of scattering on the amorphous target:

$$
\sigma=\frac{13.6 \sqrt{l_{t} / L_{r}} \cdot\left[1+0.038 \cdot \ln \left(l_{t} / L_{r}\right)\right]}{E_{0} \gamma \sqrt{1-\gamma^{-2}}}[\text { Mpad }],
$$

where $\mathrm{L}_{\mathrm{r}}$ is the radiation length, $\mathrm{E}_{0}$ is the rest energy of the particle in a GeV, $\gamma$ is the relativistic parameter. In case of use the targets for extraction of the relativistic protons: $\gamma>1$ and $D \approx \frac{14 \beta}{\gamma} \sqrt{\frac{l_{t}}{L_{r}}}$. Then the losses of particles on the target due to the nuclear interactions will be defined: $\eta_{t}=1-e^{-\bar{N} l_{t} / L_{n}} \approx \bar{N} \frac{l_{t}}{L_{n}}=\frac{l_{t}}{L_{n}}\left[1+k\left(\frac{y}{D}\right)^{m}\right]$,

where $l_{t}$ is the target thickness on the beam, and $L_{n}$ is the nuclear length.

At the account of the extraction with use of the scattering target it is necessary to consider losses $\eta_{a}$ of particles deflected by the target on the large angles and lost on the collimators and other elements of the accelerator. The magnitude of such losses can be estimated by the probability formula of the accidental process:

$$
\eta_{a} \approx\left(1-\eta_{t}\right) \cdot \int_{A}^{\infty} \frac{e^{-x^{2} / 2 D^{2}}}{\sqrt{2 \pi} D} d x,
$$

where, as it is visible from Fig. $2, A^{2}=A_{m}{ }^{2}-R^{2}$. For U70 the bounding aperture makes $A_{m} \sim 30 \mathrm{~mm}(\beta \approx 25 \mathrm{~m})$. Then $A / D$ > 1 and the magnitude of this losses will be little (Fig.5 (•)). Such losses some increased due to nuclear elastic interaction of particles with the target.

The magnitude of losses on the septum can be defined from the probability of hit the particles on the septum partition. Generally amount of lost particles on the septum depends on what place on the extraction phase space the particles scattered on the positive and negative angles hit in. The most simple case for definition of losses on the septum will when a phase shift of betatron oscillations in the extraction plane between the target and septum equals $\pi / 2+\pi n$ and the betatron frequency $Q=m / 4$, where $n$ and $m$ is integers. Then the image of particles scattered by the target on the phase space of septum location will intercross its partition under right angle (Fig.2, target1,2). The particles not hit on the septum again will dissipate on the target and the process of the extraction will proceed. As have shown analytical and numerical calculations (fig.6) the losses close to minimum in these conditions.

The losses on the septum partition can be defined from the coordinate density of scattered particles hit on a septum. In case of little sizes of a beam when $R \ll D$ it is possible to consider distribution of particles on the septum how Gaussian with r.m.s $=D$ and on the thin parti- tion of septum $(\delta<<D)$ the losses will make $\eta_{s}=\frac{\delta}{\sqrt{2 \pi} D}$ and the value of total losses:

$$
\eta \approx \eta_{t}+\eta_{s} \approx \frac{l_{t}}{L_{n}}+\frac{2 \delta}{\sqrt{2 \pi} D} .
$$

That is have received a rather simple losses function depending only from one variable - target thickness and its optimum size can be found from the equation $d \eta / d l_{t}=0$. Solving which is gained:

$$
\begin{gathered}
l_{t} \approx \sqrt[3]{\frac{\delta^{2} \gamma^{2}}{2 \pi 14^{2} \beta^{2}} L_{r} L_{n}^{2}}, \quad \eta_{t 0} \approx \sqrt[3]{\frac{\delta^{2} \gamma^{2}}{2 \pi 14^{2} \beta^{2}} \frac{L_{r}}{L_{n}}}, \\
\eta_{s 0}=2 \eta_{t 0} \quad u \quad \eta_{\min 0} \approx 3 \eta_{t 0} .
\end{gathered}
$$

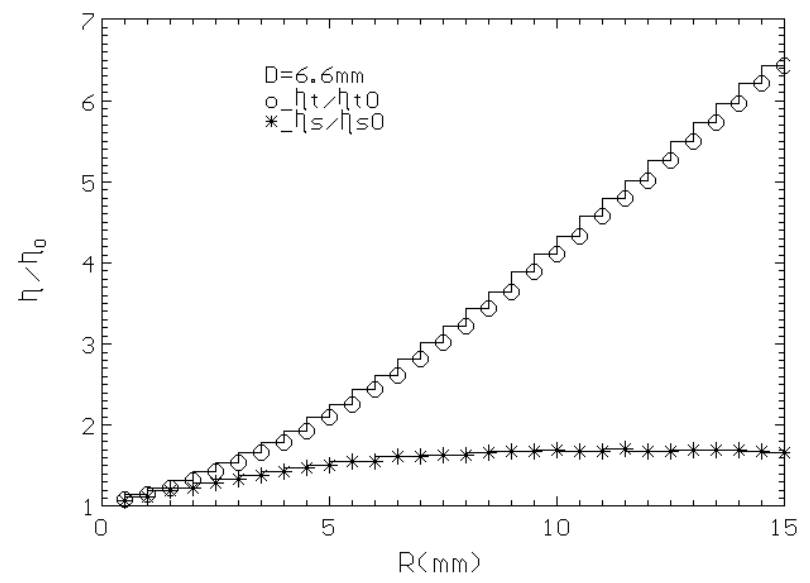

Fig.3: Dependence of relative losses versus the septum displacement from the equilibrium orbit.

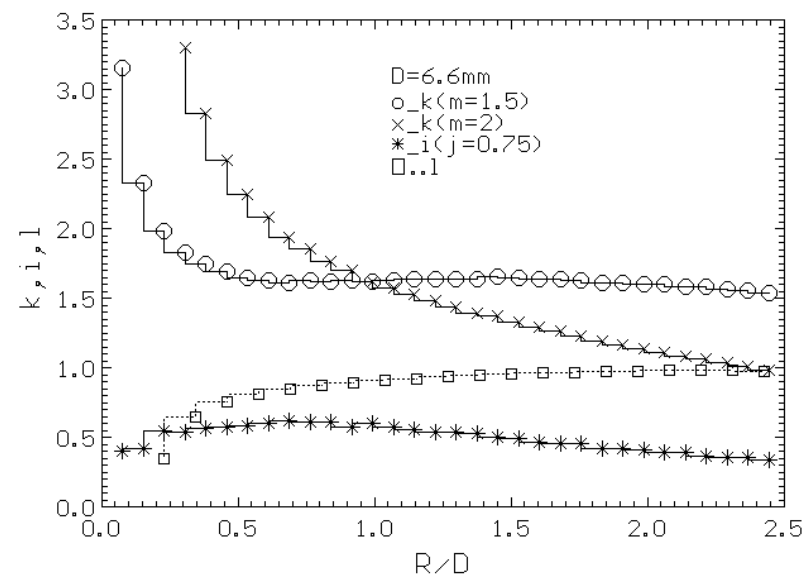

Fig.4: Dependence of approximation coefficients versus the septum displacement.

With consideration of concrete beam sizes the losses on the target and septum partition will increase (Fig.3), but the optimum target thickness (Fig.5) can be kept abovestated and then the losses will be defined:

$\eta \approx \eta_{t 0}\left[1+k\left(\frac{R}{D}\right)^{m}\right]+\eta_{s 0}\left[1+i\left(\frac{R}{D}\right)^{i}\right]=\eta_{t 0}\left[3+k\left(\frac{R}{D}\right)^{m}+2 i\left(\frac{R}{D}\right)^{i}\right]$.

For viewed forming and extraction systems the value $\mathrm{D}$ at which will be minimum losses lies in field $0.5<R / D<2.5$ and with precision in some percents the functions of 
losses on the target and septum approximate by the coefficients $\mathrm{k}=1.7, \mathrm{~m}=1.5$ and $\mathrm{i}=0.6, \mathrm{j}=0.75$. At the large size of beam $R / D>3$ the coefficients accept reference values of a diffusion process $\mathrm{k}=1, \mathrm{~m}=2$ (fig. $4(\mathrm{x})$ ). And at the finally we may done estimation of losses for all extracted beam $\eta \approx \eta_{t 0^{\circ}}(3+l \cdot \mathrm{R} / \mathrm{D})$, where $l \sim 1$ (Fig.4 ( )) and $\mathrm{R}$ is the beam size. From the losses dependencies it is visible, that in this case the total losses decrease with approaching septum to the beam and will be minimum at the $r=R$. With consider that beam size $r \approx \sqrt{\varepsilon \cdot \beta / \gamma}$, where $\varepsilon$ is invariant emittance, the value $\frac{R}{D}=0.23 \cdot \frac{\sqrt{\varepsilon}}{\sqrt[3]{\delta}} \cdot \sqrt[3]{\frac{L_{r}}{L_{n}}} \cdot \sqrt[6]{\frac{\gamma}{\beta}}$. That is at increasing energy of particles the influence of the beam size on the losses slowly raising.

In table 1 the significances of radiation and nuclear lengths for different substances are indicated, from which it is visible, that the least ratio $L_{r} / L_{n}$ have the substances with large nuclear number. So the total losses in the extraction system using as the scattering target $\mathrm{W}$ will be in $\sim 3$ times less than with the target from Be.

Table 1:

\begin{tabular}{|l|l|l|l|l|l|l|}
\hline & $\mathrm{Be}$ & $\mathrm{C}$ & $\mathrm{Al}$ & $\mathrm{Fe}$ & $\mathrm{Cu}$ & $\mathrm{W}$ \\
\hline$L_{r}, \mathrm{~mm}$ & 353 & 188 & 89 & 17.6 & 14.3 & 3.5 \\
\hline$L_{n}, \mathrm{~mm}$ & 407 & 400 & 394 & 168 & 151 & 96 \\
\hline$L_{r} / L_{n}$ & 0.87 & 0.45 & 0.24 & 0.10 & 0.095 & 0.036 \\
\hline
\end{tabular}

Table 2:

\begin{tabular}{|l|l|l|l|l|l|l|l|l|}
\hline & $\gamma$ & $\beta$ & $\mathrm{r}$ & $\sigma$ & $l_{t}$ & $\eta_{0}$ & $\eta_{1}$ & $\eta_{\mathrm{si}}$ \\
\hline & & $\mathrm{m}$ & $\mathrm{mm}$ & $\mathrm{mm}$ & $\mathrm{mm}$ & $\%$ & $\%$ & $\%$ \\
\hline $\mathrm{U} 70$ & 70 & 25 & 6 & 1 & 6 & 19 & 25 & 26 \\
\hline $\mathrm{U} 600$ & 600 & 380 & 10 & 0.2 & 1.4 & 5 & 9 & 9 \\
\hline
\end{tabular}

In the Fig.5 are shown the dependencies of losses versus target thickness obtained by computer simulation. Optimum thickness of the tungsten target and appropriate efficiency for U70 and U600 designed analytically and obtained by computer simulation are represented in table 2 .

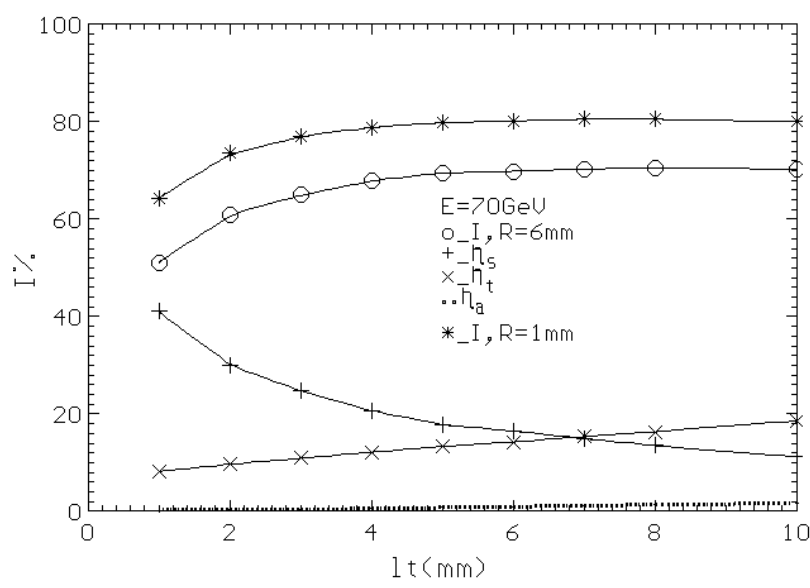

Fig.5: Dependence of losses versus the target thickness.

The total magnitudes of losses defined analytically from (3) and numerically with consider Gaussian beam density from (5) are shown with indexes (0) and (1) accordingly.

For the optimum target and septum location on the azimuth and choice the betatron frequencies are necessary to consider on the phase space how particles scattered by the target on positive and negative angles $\eta=\eta_{s+}+\eta_{s}$ hit on the septum partition. In Fig.6 are indicated the dependence of the extraction efficiency versus of betatron oscillations frequency in U70 at the use of the tungsten target with the thickness $l_{t}=6 \mathrm{~mm}$, obtained in computer simulation by the program "SCRAPER" /3/. Near to resonance lines $Q_{r}=m / 3$, where $m$ is integer, the reducing of the extraction efficiency is observed.

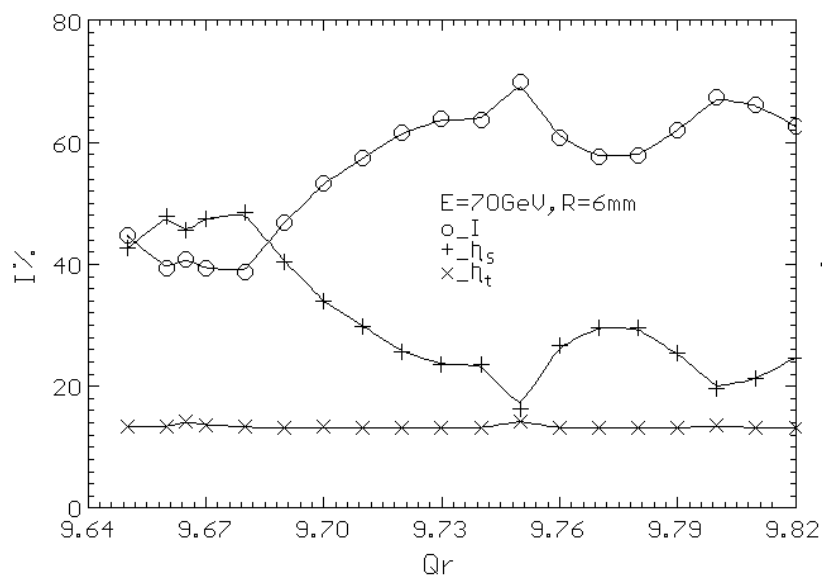

Fig.6: Dependence of the extraction efficiency and losses versus the betatron frequency.

\section{Conclusions}

From the table and expressions of losses it is visible, that the losses of particles at the extraction on the scattering target will increase with magnification of energy and reduce with magnification of $\beta$-function and diminution of a septum partition thickness. In modern developed circle accelerators as UNK, CERN for the extraction of particles the special insertions with large magnitudes $\beta$-functions $1000 \mathrm{~m}$ are provided. The modern electrostatic deflectors have a thickness up to $0.1 \mathrm{~mm}$ (that is effective thickness $\delta \sim 0.2 \mathrm{~mm})$. Then the minimum losses if extracted all beam $\left(\varepsilon=2 \cdot 10^{-4} \mathrm{~m} \cdot \mathrm{rad}\right)$ with the use of tungsten target for crude estimation will be $\eta \sim 5 E^{3 / 4}$, where $E$ is the energy of particles in $\mathrm{TeV}$. That is for energy $3 \mathrm{TeV}$, as in UNK project, when we will use such extraction is possible to receive extraction efficiency $\sim 90 \%$, and at the energies 8 $\mathrm{TeV} \sim 75 \%$. That is such method of extraction can be considered as alternate for the solution of some problems at the high energies on modern circle accelerators. In particular in the collider mode the extraction on the amorphous target can be used as additional, cutting the halo beam for parallel experiment. At the appropriate construction the target can scatter significant intensity of the beam $\sim 5 \cdot 10^{12}$ particles in the second $/ 4 /$.

\section{References:}

1. A.V.Maksimov, "Slow extraction from the U70 at the scattering on the inner targets ", Preprint IHEP 94-38, Protvino, 1994.

2. Ch.Steinbach "Slow extraction by target scattering", CERN/MPS/CO 70-7, 1970.

3. I.I.Degtyarev, et al., "Beam losses simulation in accelerators with extended version of the RTS\&T code." HEACC 98, Dubna, 1998.

4. I.I.Degtyarev, A.E.Lokhovitskii, Yu.S.Fedotov, I.A.Yazynin, "Substance choice of the scraper system elements", PAC 1997. 